\title{
Del hierro al hormigón armado en la construcción civil e industrial en Argentina
}

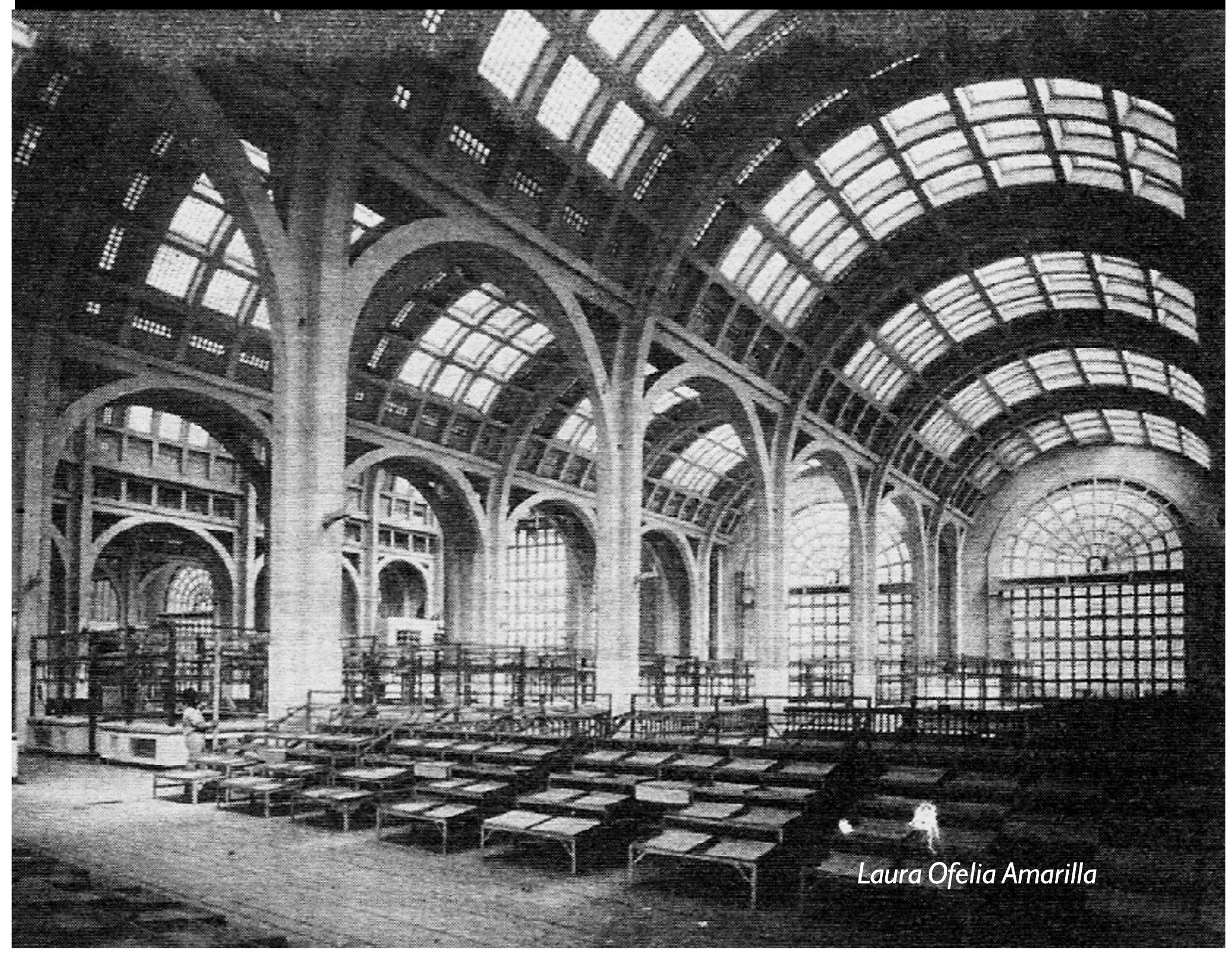


La difusión del hierro como material de construcción y las tipologías industriales legadas.

En las primeras décadas del siglo XIX, mientras Europa recibía las innovaciones de la revolución industrial, los habitantes del Virreynato del Río de La Plata se encontraban luchando por su independencia de España'. Una vez obtenida ésta, las luchas internas por el manejo del poder y las diferentes ideas sobre la conformación de la naciente nación serán un freno concreto para el desarrollo económico, y todo lo que éste puede traer consigo ${ }^{2}$. Es así que durante la primera mitad del siglo la ausencia de políticas con miras al desarrollo, la escasa población en estos vastos territorios -que se traducía en un mercado interno reducido-, la limitación en las comunicaciones -por la lejanía y los costos que esto suponía-, hacía del incipiente país emancipado un lugar anclado en el pasado.

En las primeras décadas de la segunda mitad del siglo XIX, durante el período conocido como de Reorganización Nacional, se comenzó paulatinamente a introducir algunos adelantos industriales traídos principalmente desde Inglaterra, Francia y Alemania ${ }^{3}$. Si bien la energía de vapor había sido introducida ya en la década de 1840 en Buenos Aires ${ }^{4}$, había sido un hecho puntual ya que todavía no estaban dadas las condiciones para un eficiente desarrollo industrial.

En las últimas décadas del siglo XIX la situación cambió verdaderamente, cuando se decidieron políticas tendientes a poblar y trabajar el suelo mediante la introducción de mano de obra extranjera ${ }^{5}$. Las miles de personas que arribaron desde toda Europa trajeron su empuje y sus necesidades, y si bien se dispersaron por todo el territorio muchos eligieron las ciudades más grandes, como Buenos Aires, Rosario y Córdoba, como lugar de asentamiento ${ }^{6}$. Los inmigrantes rápidamente se organizaron en colectividades y buscaron sumar su fuerza laboral a la existente en el país. En ese contexto, algunos de ellos instalaron talleres artesanales en los que se comenzó a producir todo tipo de elementos y artefactos para la vida diaria?.

Interesa aquí la difusión del hierro en la construcción en Argentina por esta época; debemos entonces subrayar que en estos lejanos inicios hubo dos momentos que vale la pena señalar: por una parte la importación completa de estructuras metálicas para armar en el sitio y, por otra, la importación de hierro en barras y en láminas que luego se trabajaban en los talleres y fundiciones, hasta que se inició la producción de hierro, ya avanzado el siglo XX. Los avances de Louis-Marie Navier, en 1826, y el descubrimiento de A. Darby de la fundición del hierro forjado, eran conocidos por los inmigrantes que montaron los pequeños talleres ya mencionados, fundiciones y herrerías, quienes sin embargo dependían de hierro importado para la producción de sus artículos, ya que no estaban en condiciones de producir el metal. Ellos satisfacían las demandas crecientes de una población cada vez mayor, donde se comenzaron a producir artefactos de hierro como camas, campanas, cocinas (a leña), máquinas de amasar y mezcladoras, calderas, y muy especialmente, todo tipo de piezas para ser empleadas en la construcción, desde bulones, clavos, remaches, alambres, cadenas, artefactos para la iluminación ornamental de las ciudades, rejas, tubos de hierro para ensamblar diferentes tipologías constructivas, piezas para los servicios sanitarios ${ }^{8}$, hasta estructuras completas en hierro para la construcción de edificios, cuando los talleres empezaron a ampliar y diversificar su producción transformándose en talleres mecánicos y metalúrgicos?.

Junto con la introducción de nuevos pobladores, los gobiernos del momento incentivaron el desarrollo de las ciudades consideradas más importantes, invirtiendo en obras públicas que serían las sedes de los poderes de estado y los diferentes ámbitos funcionales para el desenvolvimiento de la administración pública, de salud, de educación, de justicia y obras de infraestructura necesarias para dotar a las ciudades de servicios de salubridad, como el agua corriente y las cloacas. Asimismo, conexiones como puentes peatonales, vehiculares $y$, posteriormente, para el paso del tren, que se convertiría en lazo de las comunicaciones y del transporte de mercaderías, sin descuidar las obras de ornato $^{10}$. Los esquemas tipológicos utilizados como base para estas arquitecturas oficiales, y también para las privadas, fueron las ya probadas en el extranjero", como así también las estructuras prefabricadas, que en un primer momento fueron extranjeras ${ }^{12}$, igual que los profesionales ${ }^{13}$ y la mano de obra capaz de concretar estas nuevas construcciones ${ }^{14}$. Argentina no contaba, a diferencia de Italia, con una historia arquitectónico-

Foto pág. opuesta: José $L$. Delpini, arquitectos Viktor Suscic y Raúl Bés (1929-

1934).

Mercado de

Abasto de Buenos Aires. Interior 
urbana, -que también poseían los países europeos como Francia, Inglaterra y Alemania-, por entonces el país tenía hacia atrás las tipologías legadas por la Colonia y hacia adelante todo por hacer, inclinándose autoridades e inversores rápidamente por los lineamientos y normas europeas de la Escuela de Bellas Artes de París, siempre dentro de lo que podríamos llamar el Eclecticismo Historicista, en cuanto al carácter y la representación que satisfacía los sueños de los gobernantes del momento ${ }^{15}$. Así como en Europa se adoptó como «principio constructivo» la estructura de esqueleto para las grandes obras del estado y privadas, como sostiene Renato de Fusco ${ }^{16}$, también en nuestro país se utilizó el mismo principio para edificios del estado, mercados, estaciones de trenes, pasajes comerciales, etc., mientras que para el caso de las obras domésticas o de menor envergadura se utilizó piezas o partes de estructuras metálicas, como las columnas de hierro en los patios de luz y las galerías, las vigas de hierro como sostén de bovedillas en las cubiertas, así como fachadas completas para comercio, estructuradas con vidrieras y cortinas de enrollar. Asimismo se manifiestó con fuerza significativa en el campo constructivo de la cultura argentina del siglo XIX, la «arquitectura de la ingeniería», -en el sentido que le confiere a la frase De Fusco-, realizada en muchas ocasiones por ingenieros.

Los profesionales que actuaron en el medio por entonces, la mayoría extranjeros, a los que se irán sumando paulatinamente los nativos que estudiaron fuera y los primeros que se gradúan en Argentina ${ }^{17}$, buscaron representar con sus arquitecturas el espíritu argentino que pensaban podía representar el nuevo país, logrando en definitiva una suerte de «clave argentina» $y$, si bien trabajaron sobre esquemas tipológicos extranjeros ya probados, y en algunos casos con materiales importados del extranjero, puede decirse que la «clave» es el carácter completo conferido a tales arquitecturas. Carácter en cuanto a la fuerza simbólica que se imprime a la representación ${ }^{18}$ y en cuanto al uso que se hace del material de hierro. La innovación constructiva implicaba un sistema de ensamblaje del hierro fundido que permitía el armado eficaz, rápido, a menor costo y de mayor eficiencia en relación a los incendios, al contrario de la madera, lo que fue utilizado eficazmente introduciendo los cambios necesarios cuando era menester ${ }^{19}$.

Precisamente la introducción del sistema innovador para la producción de estructuras de hierro fundido posibles de ser ensambladas, posibilitó mayor rapidez en su elaboración y reducción de costos, lo que se revirtió en la posibilidad de mayor uso de todos los elementos propuestos en hierro para la construcción, tanto los de tipo estructural, como los de tipo decorativo ${ }^{20}$. Así, las fábricas de entonces proponían en sus catálogos y propagandas productos completos a ser montados rápidamente, como retretas, viveros, invernaderos, galpones de diverso tipo y para diverso uso, sistemas completos para pabellones expositivos, etc. Inclusive se debe tener en cuenta que se utilizó estructuras completas para grandes edificios privados y para comercialización de productos, como ya se mencionó.

Estas estructuras en hierro fueron un principio constructivo tomado por los proyectistas en Argentina al final del siglo $X I X$ y principios del $X X$ por las innegables posibilidades que brindaba, entre ellas la gran espacialidad interior que permitía, la luz natural que se podía obtener por medio de cerramientos vidriados, lo que implicaba espacios interiores completamente nuevos $y$, ciertamente, la rapidez constructiva necesaria en un período que podemos mencionar como fuera de límites por la cantidad de obras que se realizaron. Es entonces evidente que la transformación en los métodos del trabajar el hierro, la incorporación del hierro nunca antes empleado de ese modo, así como su tratamiento y ensamblaje, colaboró en la organización de la industria de la construcción-industrialización de la totalidad de los componentes de muchas tipologías como las mencionadas y otras unidades similares, incidiendo fuertemente en el desarrollo de las ciudades, que modificaron su edilicia en corto tiempo.

\section{La difusión del hormigón armado como material de construcción y las tipologías legadas.}

En el mismo tiempo histórico en que se construía una cantidad importante de edificios públicos y privados utilizando el hierro como material estructural: fines del siglo XIX y principios del $X X$, la construcción de grandes obras de infraestructura incidió en los estudios de aplicación de un nuevo material: el hormigón armado, que se perfilaba como de múltiples capacidades para ser utilizado en la arquitectura civil. Mientras en Europa la invención y el perfeccionamiento de este material avanzaba rápidamente, fruto de la investigación tecnológica francesa de J. Monier, F. Coignet, F. Hennebique y A. de Baudot, más la tradición técnica-historiográfica de $\mathrm{E}$. Viollet Le Duc y Choisy ${ }^{21}$, en Argentina el desarrollo del producto fue más lento. En la primera etapa de este período era necesario importar los dos productos que componen el hormigón armado, hierro y cemento, ya que no eran producidos en el país, subrayando además que el traslado de ambos era costoso y que el hierro fue preferido por su ductilidad, versatilidad y por las disposiciones técnicas que se manejaba al momento de emplearlo, mientras que con el cemento había que practicar y experimentar, y por tanto fue poco empleado. Según Jorge F. Liernur, un pionero en este tema fue el ingeniero Carlos Enrique Pellegrini quien, ya en el 


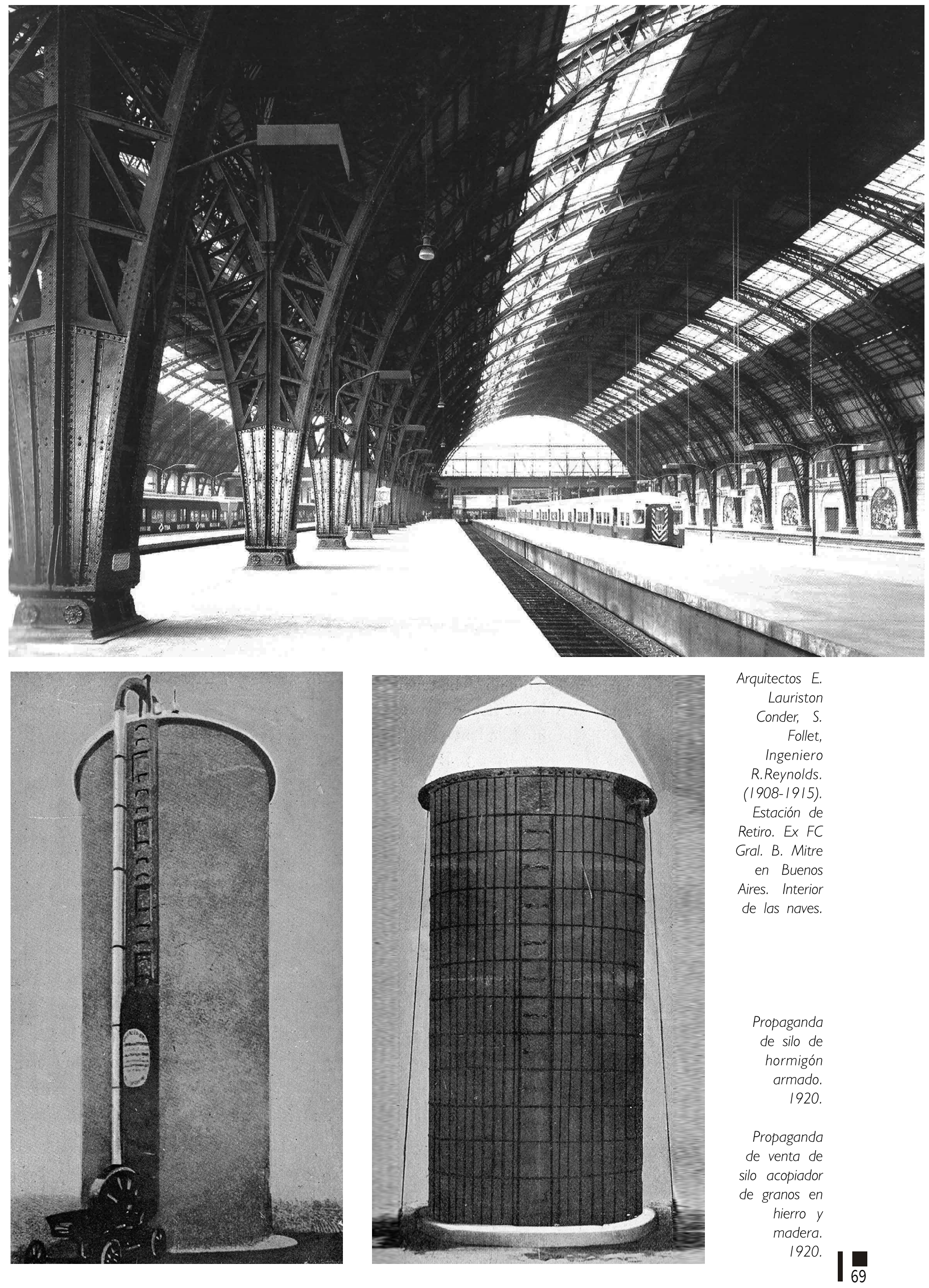




\section{LA CANTÁBRICA$$
\text { (SOCIEDAD ANÓNIMA) }
$$ \\ Capital realizado $\$ 3.000 .000 \mathrm{~m} / \mathrm{n}$.}

Grandes Talleres para la elaboración del hierro en general

Fabricación \& Importación de Artículos rurales

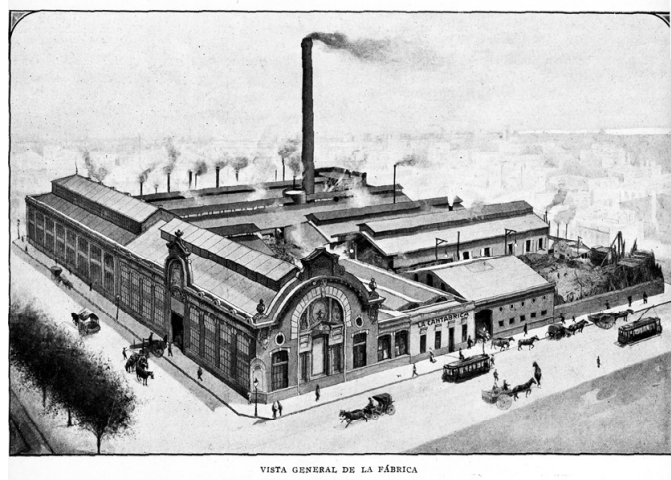

Existencia permanente y surtido completo de Postes, Alambres, Varillas, Torniquetes y toda clase de Materiales para Alambrados

Propaganda de ventas de La Cantábrica. Buenos Aires. 1920.

Propaganda de la Casa Agar Cross \& Co. Ltd. sobre

distintos implementos y construcciones en hierro. 1920. Bombas, Molinos, Tanques Australianos, Bebederos de hierro y de madera,
Represas y toda clase de Aparatos para instalación de Aguadas Arados, Rejas, Rastras y

Bretes, Tranqueras, Gaipones y toda clase de Trabajos de earpinteria Rural Bretes, Tranqueras, Gaipones y toda clase de Trabajos de earpinteria Rural
Fundición, Construcción y Reparación de Maquinarias. - eonstrucciones Metálicas PROYECTOS PRESUPUESTOS

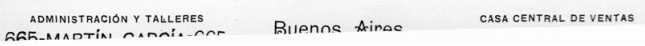

lejano año de 1854, fue uno de los primeros en proponer el uso de la «piedra de sillería artificial»»22, quedando su prédica en éste y otros temas sin ser tomada en cuenta. Hubo que esperar unas décadas para que el tema volviera a surgir. En un caso en la Provincia de Córdoba, con la fábrica de cales hidráulicas «La Argentina», fundada por el Dr. Bialet Massé en 1884, la que fue montada con el objetivo específico de construir el primer dique en el Lago San Roque de la misma Provincia ${ }^{23}$. El otro caso en la ciudad de Rosario, donde a instancias de Nicolás Fuhr se instaló la primera fábrica de cemento, en 1888. Se abrió luego una planta en Buenos Aires, prueba realizada por el Estado a través de la Dirección de Aguas Corrientes, Cloacas y Adoquinado, en la localidad de Barracas, estando a cargo del ingeniero inglés Bateman el proyecto y construcción de la fábrica; y posteriormente en Tandil, donde Nicolás Derossi comenzó un ensayo de fabricación en $1889^{24}$. Todas estas producciones no subsistieron por dificultades diversas, siendo una de ellas el costo del producto, mayor que el importado. Todos los ensayos y productos tuvieron control científico y pruebas con sus respectivos infor-

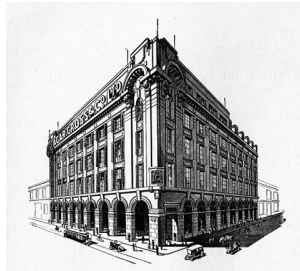

Maquinaria e Implementos para cualquier género de faen Maquinaria y Accesorios INDUSTRIALES de todas clases

\section{Maquinaria y Accesorio} ELÉETRICOS

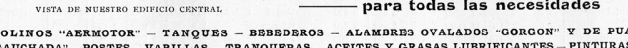

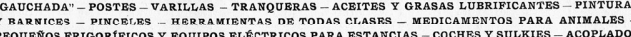

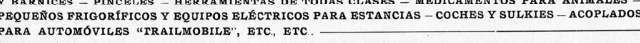

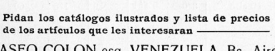

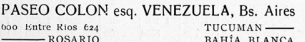
AGARCROSS $\approx \mathbf{C}_{\text {a }}^{0}$ mes técnicos publicados por la Revista «La Ingeniería» y en los Anales de la Sociedad Científica Argentina $^{25}$. Por su parte, Liernur nombra a José Moine como quien obtuvo la primera patente efectiva para producir cemento, de la original francesa, en 188926. Ya a principios del siglo XX, en 1907, se instaló la Fábrica Nacional de Cemento Pórtland en la provincia de Córdoba, en la localidad de Rodríguez del Bustos, por iniciativa de los ingenieros Senestrari y Gavier, quienes fueron asesorados por los ingenieros franceses $E$. Candiot y $M$. Chatelier ${ }^{27}$. En 1917, el ingeniero Marcelo Garlot y el sr. Pablo Verzini levantaron una fábrica en la localidad de km 7 próxima a la ciudad de Córdoba, firma que alcanzó a producir 30,000 toneladas anuales de cemento Pórtland en el año 1931, admirable logro ya que su inicio corresponde a la época de la Primera Guerra Mundial, que en esos momentos asolaba parte del mundo, no existiendo posibilidad de recibir tecnología ni equipos de Europa. Sus fundadores recurrieron, por lo tanto, a elementos locales, uno de desechos industriales y otros creados ex profeso y aplicando su propia tecnología, sentando las bases de lo que posteriormente fue la Corporación Cementera Argentina. Mientras que en 1926 se constituyó Loma Negra Cía. Industrial Argentina, en el partido de Olavarría, Provincia de Buenos Aires, con el impulso de Alfredo Fortabat, sucedió algo similar en Córdoba, cuando Juan Minetti fundó la Compañía Sud Americana de Cemento Pórtland e instala, en Dumesnil, una fábrica de cemento ${ }^{28}$

Como vemos, en base a las fechas indicadas se estaban dando los primeros pasos en la producción nacional de cemento, lo que traerá como consecuencia la sustitución del hierro por el hormigón armado como material estructural en la construcción civil, hecho concretado finalmente a partir de la década de 1930. Colaboró en la consolidación de esta producción la evolución del país en la segunda mitad del siglo XIX, más los conocimiento que llegaban a estas tierras desde Europa, y ciertamente la necesidad de hacer en corto tiempo grandes obras de infraestructura acordes con el desarrollo, como pavimentos, puentes, elevadores de granos en el puerto de Buenos Aires, diques, entubamientos, canalizaciones y líneas de subterráneo. Tales necesidades convirtieron a la administración del estado nacional en un excelente cliente para empresas extranjeras que habían comprado las patentes de Monier y de Hennebique. Es así como algunas empresas alemanas se instalaron para realizar trabajos de envergadura. La empresa del ingeniero Gustav Adolf Wayss/Freitag A.G. (que trabajó y desarrolló influencia también en Brasil y en Uruguay) comenzó a trabajar en 1909 en el puerto de Buenos Aires, mientras que Siemens Bauunion lo hizo en $1923^{29}$. 

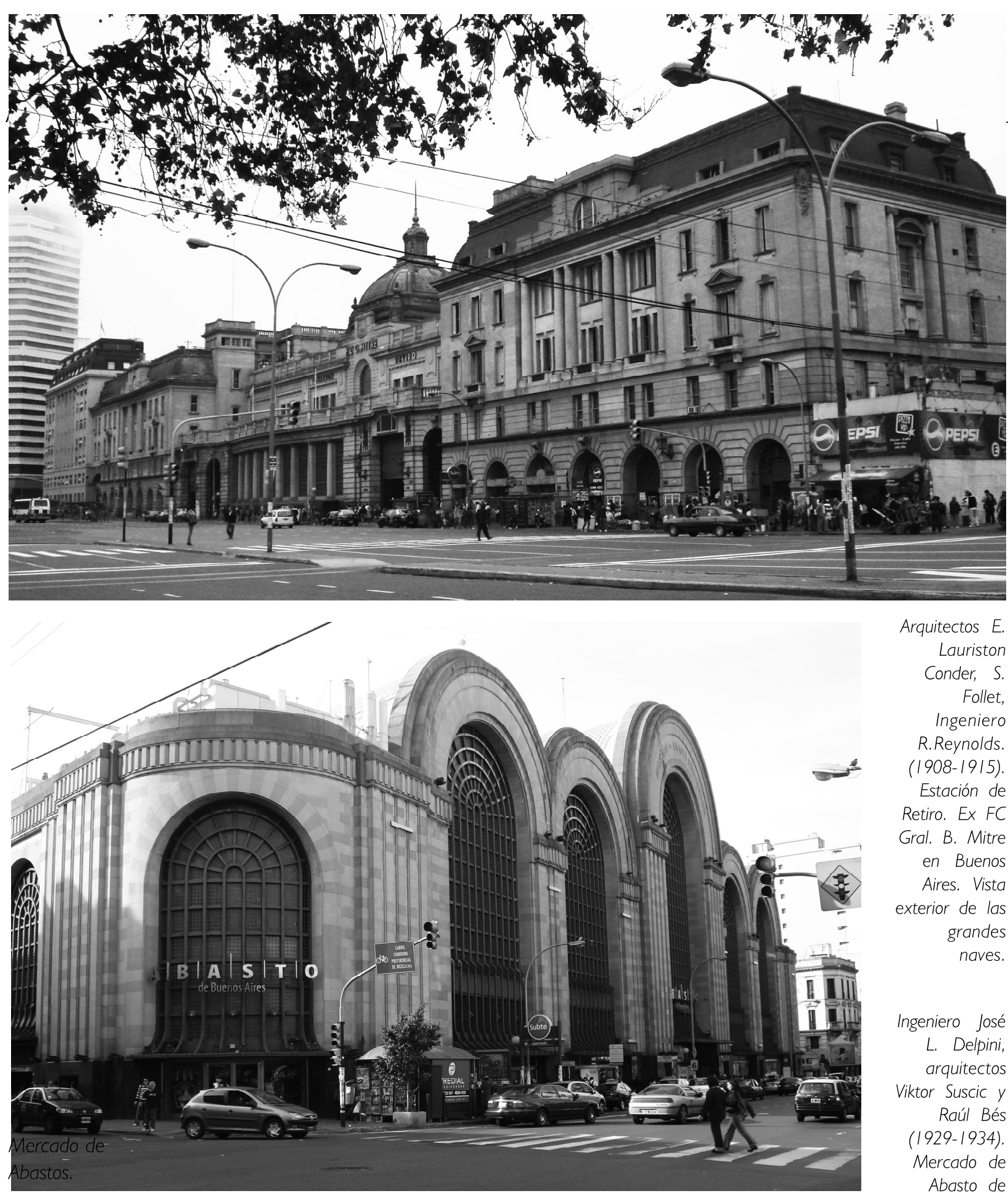

Arquitectos $E$. Lauriston Conder, S. Follet, त Ingeniero R. Reynolds. (1908-1915). Estación de Retiro. Ex FC Gral. B. Mitre en Buenos Aires. Vista exterior de las grandes naves.

Ingeniero José L. Delpini, arquitectos Viktor Suscic y Raúl Bés (1929-1934). Mercado de Abasto de Buenos Aires. Reconvertido a Centro de Compras

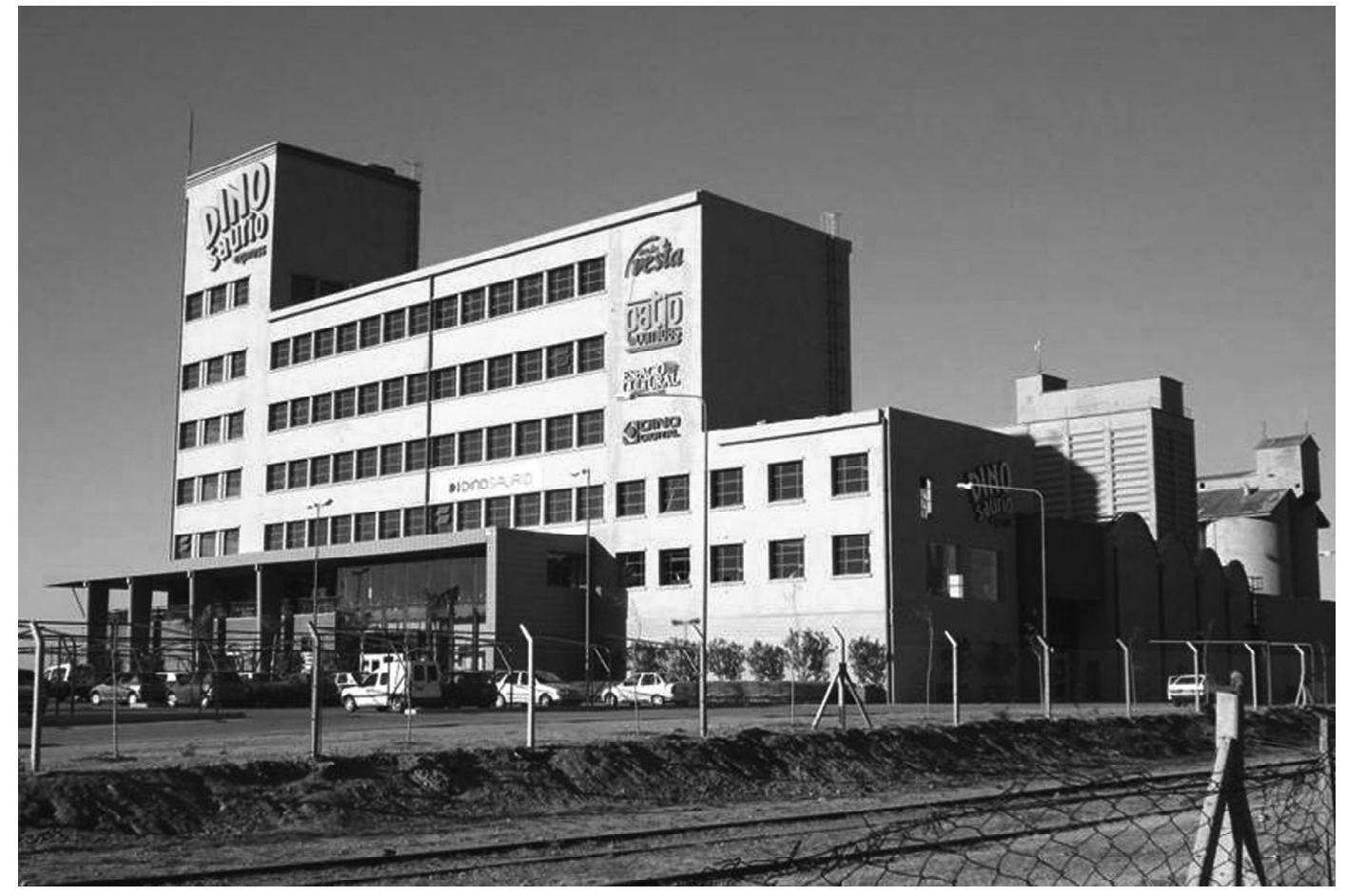

Autor no precisado (1940). Molino Letizia en la ciudad de Córdoba. Reconvertido a Supermer- 


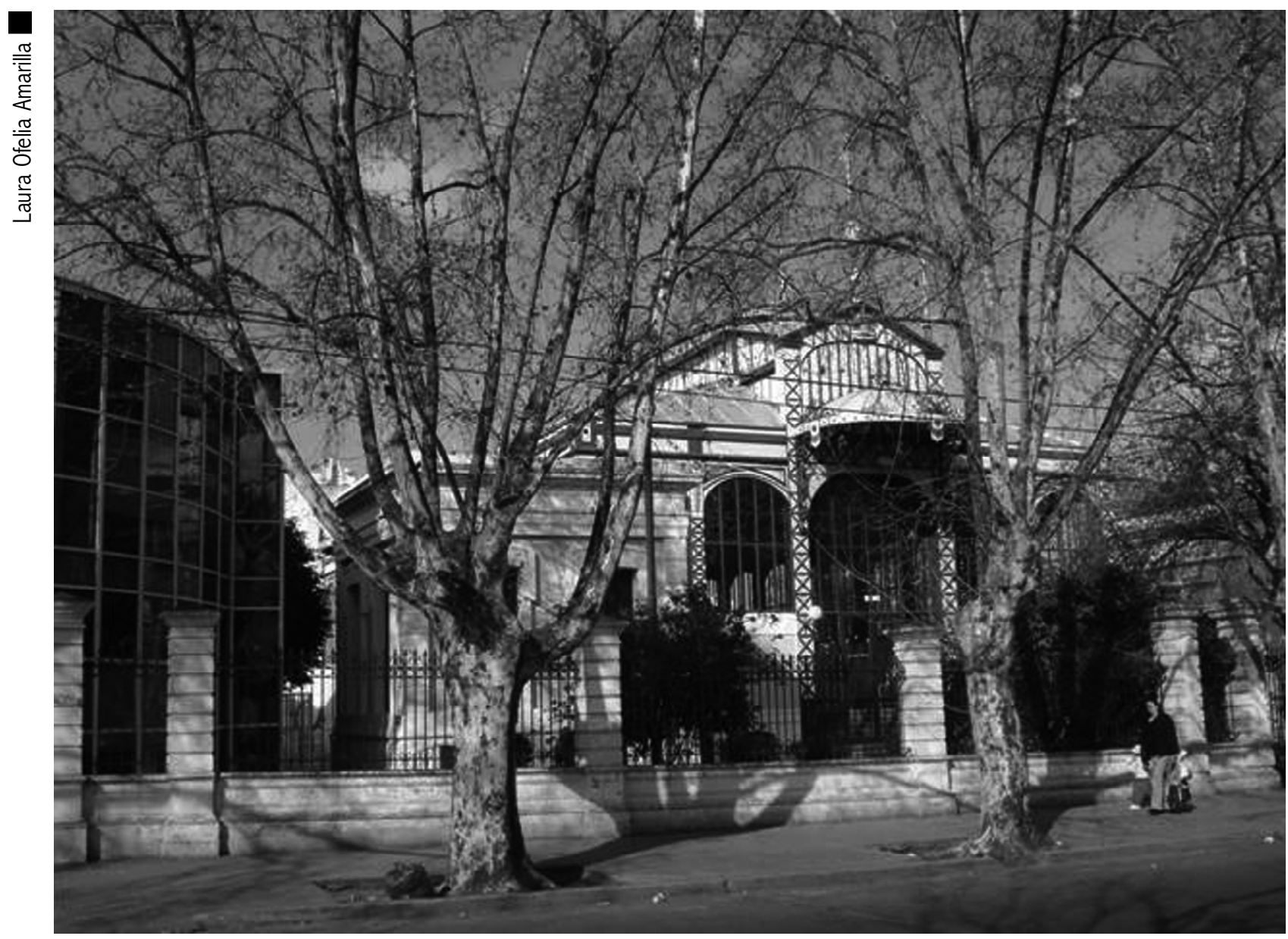

Ingeniero Baltasar Ferrer (1918). Mercado General Paz en Córdoba.
Por otra parte, el propio Hennebique, quien había desarrollado su sistema patentándolo en 1892, se encargó de popularizarlo en América Latina, demostrando con los ejemplos construidos, entre ellos su propia casa, su slogan de confiabilidad, desarrollando además normas de proyecto y ejecución que colaboraron para reglamentar el uso del hormigón en los países europeos, extendiéndose tales reglamentaciones a América Latina.

Al mismo tiempo, también en Argentina fueron realizados experimentos desde comienzos del siglo $X X$, como los del ingeniero Domingo Selva, integrante del Departamento de Obras Públicas de la Municipalidad de Buenos Aires; y de Ludovico Ivanissevich, quienes realizaron un sistema antisísmico en 1904. Si bien en la enseñanza de la Facultad de Ingeniería de Buenos Aires los cálculos eran prevalentemente de origen alemán, no se descuidaba la influencia francesa mediante la bibliografía existente que llegaba de Europa. Esto surge de la revisión de la revista «La Ingeniería», órgano oficial del Centro Argentino de Ingenieros (fundado en 1896), en cuyas páginas podemos hallar artículos referidos al cálculo en estructuras de hormigón, con referencias a fuentes bibliográficas europeas. También la revista de la Sociedad Central de Arquitectos dio lugar al nuevo material dedicando en la década de 1920 regularmente artículos destinados a divul- gar los procedimientos elementales de cálculo, diseño y ejecución de estructuras, en muchos casos bastante complejas ${ }^{30}$

Las reglamentaciones para regular las construcciones comenzaron a reflejar el uso del hormigón en precauciones contra incendios en edificios públicos y edificios residenciales, destacando, por ejemplo, que las escaleras debían construirse de hormigón armado, y que en los edificios comerciales los garajes de capacidad para más de tres coches se construirían exclusivamente con muros de mampostería y columnas, vigas y losas de hormigón armado.

Pronto fueron vistas en Argentina las ventajas que aportaba el hormigón armado cuando comenzó a ser utilizado: por una parte, la seguridad que ofrecía ante la humedad, y por otra, ante el fuego. Si bien antes de 1930 en el país no se podía dar respuesta a las necesidades cada vez mayores de cemento para el hormigón armado, éste se convirtió entonces en el más importante material estructural en la construcción civil de la primera mitad del siglo $X X$, y en la actualidad continúa siéndolo. El nuevo sistema de construcción que el hormigón posibilitó dio lugar a la evolución de las ideas constructivas, respuesta a las nuevas necesidades de modernidad, así como a características estéticas novedosas que conformaron un paisaje urbano diferente en las ciudades más populosas de la Argentina. 


\section{A modo de reflexión}

Sin duda alguna todo lo antedicho transformó radicalmente la arquitectura y el urbanismo en la Argentina, trayendo consigo una serie de cambios importantes para la vida de los ciudadanos de nuestro país. Primero fue el hierro posibilitando la infraestructura y, junto a ésta, las grandes construcciones para la higiene y salu- bridad de una población que creció exponencialmente, así como las sedes de la nueva república. Vendrían acompañando las viviendas de inquilinato para los operarios de las fábricas y los contendores para la producción en serie propia de la industria, y todo lo que conlleva. Con pocos años de diferencia, el otro material que se introdujo, el hormigón armado, provocó nuevamente un vuelco en las construcciones tanto civiles como industriales. Los resultados de la introducción de ambos materiales y sistemas constructivos usados están a la vista y pueden ser analizados a la luz de las pautas actuales, considerando siempre que fueron realizaciones de otras épocas y con otras ideas.
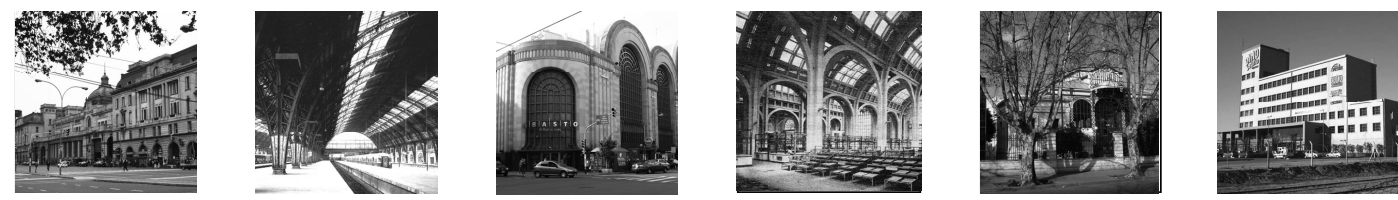

Notas

* Este trabajo corresponde al Capítulo I de mi autoría, publicado en la siguiente obra; Manuela Mattone; Laura Amarilla, Architettura in ferro e calcestruzzo armato. Nuove tecnologie costruttive tra Ottocento e Novecento in Italia e in Argentina, CELID, Torino, 20ll.

I El inicio de la emancipación de las colonias del Río de la Plata se produjo en 1810 , cuando la llamada Junta Patria destituyó del gobierno de Buenos Aires al Virrey Cisneros. Proceso que continuó por varios años y con diferentes gobiernos.

2 Las luchas internas de las provincias por el poder se prolongaron hasta finales de la década de 1850, cuando es nombrado Presidente de la Nación Argentina Bartolomé Mitre.

3 En la década de 1860 algunos emprendedores habían instalado establecimientos en los que se procesaban productos alimenticios, principalmente.

4 Jorge Tartarini (Investigación y textos), Guía patrimonio cultural de Buenos Aires. 6. Arquitectura industrial, Dirección General de Patrimonio, Buenos Aires, 2006 p. 18.

5 Los gobernantes del joven país querían que éste se pareciera más a Europa. Para lograr este objetivo, instrumentaron una política liberal y cosmopolita, importando capitales, cultura, y hasta las personas capaces de poblar la nación. Para ello el Presidente Nicolás Avellaneda promulgó una Ley de Inmigración (19 de octubre de 1876) que buscó hacer realidad el con- cepto de Alberdi, «gobernar es poblar».

6 Según los censos nacionales, para 1869, Buenos Aires contaba con 187.100 habitantes, mientras que en 1914 la población era de 1.575.800; en Córdoba, para las mismas fechas, las cifras son de 29.000 a 122.000; en Rosario, de 23.000 se pasó a 226.000, mientras que en el caso de La Plata, ciudad fundada en 1882, para 19|4 la población era de 101.000 habitantes.

7 Para el año 1895, según el censo Nacional, los talleres artesanales pertenecían, en su mayor parte, a inmigrantes. Ver en José Panettieri, Sintesis histórica del desarrollo industrial argentino, Macchi, 1969, p.29.

8 Los talleres de entonces en la ciudad de Buenos Aires, Ilamados fundiciones, que producían todo tipo de artefactos, como los mencionados, eran los de las firmas Vasena, Ottonello, Zamboni, Rezzonico y Merlin. Ver en Jorge Liernur, Arquitectura en la Argentina del siglo XX: La construcción de la modernidad, Fondo Nacional de las Artes, Buenos Aires, 2001, p. 86; Jorge Tartarini, op. cit., p. 17

9 Muchas obras de envergadura sumamente importantes para la época se construyeron con el sistema de ensamblaje de hierros en la nación, y como ejemplo señalamos una obra emblemática, como el Hotel Plaza frente a la Plaza San Martín en la ciudad de Buenos Aires, inaugurado el 15 de julio de 1909 . Construido sobre una estructura de hierro fabricada completa- mente en Argentina, juntamente con muros de ladrillos de máquina, cuya fábrica se instaló para hacer la obra. Ver en María Susana Azzi; Ricardo De Titto, Pioneros de la industria argentina, ILSHA, Editorial El Ateneo, Buenos Aires, 2008, pp. 108-109.

10 En las ciudades más importantes del país, Buenos Aires, Rosario y Córdoba, se construyeron en pocas décadas, entre 1890-1920, palacios, viviendas en altura para renta, palacios de justicia, casa de gobierno, hospitales, teatros, iglesias, escuelas, cárceles, mercados, galerías comerciales, las dependencias de los puertos de Buenos Aires y Rosario, los silos contenedores, las estaciones y su equipamiento para el ferrocarril, así como todo tipo de obras de ornato y embellecimiento.

I I Jorge F. Liernur sostiene que en cuanto a las tipologías a emplear en la construcción pocos son los interrogantes que se les plantearon a los que trabajaban en ese campo ... «para quienes se desempeñaban en nuestro país se presentaron muy pocos interrogantes inéditos, o al menos no explorados hasta entonces, en el orden de la creación tipológica, pero en general, estos parecen haber incidido débilmente en la formación de nuestra particularidad»... La única tipología característica de la Argentina moderna, y poco frecuente en otros países, sería la casa popular de patio lateral. Ver en lorge $F$ Liernur, op. cit., p. 41

12 Un caso es el del Pabellón Argentino para la Exposición de 
París de 1889, que fue fabricado en hierro en Francia, siendo posteriormente trasladado y montado en la Plaza San Martín de la ciudad de Buenos Aires, destinándolo a ser sede del Museo de Bellas Artes. Ver en Jorge Liernur, op. cit., pp.89-90

13 Según los censos de 1895 y 19|4, las cifras de profesionales de la construcción que se desempeñaban en el país eran: en 1895, 396 arquitectos y 1.481 ingenieros. Para 19/4, 4.746 en total; de ellos 2.798 constructores, 1.404 ingenieros y 623 arquitectos. Ver en Jorge Liernur, op. cit., p.38.

I 4 La mano de obra que se empleó en estas construcciones mayoritariamente fue de los inmigrantes, donde sobresalieron los italianos precisamente por provenir de una región con gran prestigio constructivo.

I 5 El «eclecticismo historicista» fue el lenguaje utilizado para mediatizar el carácter representativo simbólico en obras tanto públicas como privadas, realizadas en este período.

16 Renato De Fusco sostiene que ya en 1780 empiezan a usarse las columnas de fundición en el interior de las hilanderías, progresando hasta adoptar como «principio constructivo» la estructura de esqueleto de hierro y vidrio ensamblada. Ver en Renato de Fusco, Historia de la arquitectura contemporánea, Celeste Ediciones, Madrid, 1992, pp. 30-32.

17 Se señala algunos; italianos: Tamburini, Pellegrini, Fossatti, Moneta, Canepa, Cometa, Canale, Bettolli; franceses: Le Monnier Dormal, Benoit; alemanes: Altgelt, von Arnim, Buttner, Moog. Nativos que estudiaron en Europa: J. Buschiazzo, Ernesto Bunge, Héctor Ayerza, entre otros. También se contrataba a profesionales que tenían su escritorio en Europa o EEUU., como René Sargent, Louis Sortais, Gustavo Heine, entre otros.

18 Los edificios del estado adquieren de acuerdo a esto una fuerza simbólica que representa a un estado nuevo, pero con raíces en Occidente y deben éstas ser visibles tanto en el exterior como en interior.

19 Técnicamente los profesionales que actuaron en este período adoptaron lo ya probado, sólo que el nuevo material y el sistema innovador de ensamblaje requería de pericia suficiente, así como de adaptaciones, en base a la monumentalidad del edificio o a su complejidad.

20 Ramón Gutiérrez, «Los italianos en la transformación tecnológica de la construcción», en Graciela Viñuales, Italianos en la arquitectura argentina, Cedodal, Buenos Aires, 2004, pp. 99-106; Graciela Viñuales, «Del Ticino a la Argentina: los Righetti en la construcción», op. cit, pp. 123.

21 Renato de Fusco, op.cit., p. 173

22 Jorge Liernur, op.cit., p. 82.

23 Laura Amarilla, "Patrimonio industrial como recurso de turismo cultural en la Provincia de Córdoba», en Miguel Ángel Álvarez Areces; Jorge Tartarini (Coordinadores), Patrimonio Industrial en Iberoamérica. Testimonios de la memoria del trabajo y la producción, AySA, Agua y Saneamientos Argentinos. Asociación de Arqueología Industrial. Industria, Cultura, Naturaleza. INCUNA, Gijón, España. Gobierno del Principado de Asturias, p.86; Ver en Norberto Emundo Huber; Sergio Gabriel Capdevila, «Cal hidráulica de Bialet Massé. Su vigencia. Uso de la cal hidráulica en la restauración de edificios históricos», en La dimensión social del patrimonio. Enfoque. Teorías. Intervenciones. Técnicas. Artes. Patrimonio inmaterial. VIII Congreso Internacional de Rehabilitación del $\mathrm{Pa}$ trimonio Arquitectónico y Edificación, Centro Internacional para la Conservación del Patrimonio, Buenos Aires, 2006.

24 Cfr. Registros de la Sociedad de Cemento Pórtland de Argentina.

25 Las virtudes del cemento argentino fueron defendidas desde el inicio de su producción, como se puede ver en los puntos siguientes: a) En este sentido, en el diario «Tribuna Nacional» de Enero de 1885, figura el siguiente comentario: "Conviene advertir que la experiencia del "cimento» argentino a pesar de la mala voluntad manifiesta contra él, dieron siempre, casi sin excepción, resultados ventajosos a su favor. Era mejor su calidad, mayor su resistencia, que la del «cimento» inglés, sólo que el costo resultaba un $20 \%$ más caro». Los seño- res M. Puiggari y Luis Silveyra realizaron un estudio de este cemento, que figura citado en la publicación de Marcial R. Candiotti en la Revista del Archivo de la Sociedad Científica Argentina 1876. b) Para la fábrica de Bialet Masse, realizó un informe técnico el Ing. Jorge Duclout, publicado en la revista «La Ingeniería - TI, año 1887, bajo e título «Ensayo de las cales y cementos de Córdoba», donde analiza las características de los cementos producidos por la fábrica, cuyos análisis químicos fueron hechos por el Dr. Kyle. c) Para la fábrica de Derossi el estudio del producto logrado lo realizó el Dr. Anastasio Quiroga y se publicó bajo el título «Informe sobre el Cemento Argentino». Anales de la Sociedad Científica Argentina -T. XXVIII. d) Para la Compañía Argentina de Cemento Pórtland, que en noviembre del mismo año colocó la piedra fundamental de la que sería posteriormente la fábrica de cemento «San Martín», fue el Dr. Abel Sánchez Díaz quien defendió la calidad de este producto nacional y el 30 de junio de 1919 en el Centro Argentino de Ingenieros, con el auspicio de la Sociedad Química Argentina, pronunció una conferencia en la que presentó el flamante cemento portland «San Martín». La experiencia llevada adelante con todas estas pruebas demostró que el producto argentino era de buena calidad.

26 Jorge F. Liernur, op.cit., pp. 82 84.

27 Carlos Duvoy, La evolución de la industria argentina del cemento pórtland, Buenos Aires, Instituto del Cemento Pórtland Argentino, 1972; Marcelo Garlot, «La industria del cemento», en Magazine Geográfico Argentino, Buenos Aires, Año I No 6, diciembre 1933, p.l05 y ss.; Cfr. Registros de la Sociedad de Cemento Pórtland de Argentina.

$28 \mathrm{Idem}$.

29 Jorge Liernur, op. cit., p. 82-84.

30 Eduardo César Gentile, «Acero, hormigón y acero. Seis décadas de diseño y representación de sistema de esqueleto estructural en la Argentina 1930-1990". En Actas del $X$ Congreso Internacional de Ingeniería Gráfica, Málaga, España, 1998 